\title{
SAXS study on the morphology of etched and un-etched ion tracks in apatite
}

\author{
A. Nadzri ${ }^{1, \text { a }}$, D. Schauries ${ }^{1}$, B. Afra ${ }^{1}$, M. D. Rodriguez ${ }^{1}$, P. Mota-Santiago ${ }^{1}$, S. Muradoglu ${ }^{1}$, A. Hawley ${ }^{2}$ and P. Kluth $^{1}$ \\ ${ }^{1}$ Department of Electronic Materials Engineering, Research School of Physics and Engineering, Australian National \\ University, ACT 2601, Australia \\ ${ }^{2}$ Australian Synchrotron, 800 Blackburn Road, Clayton, VIC 3168, Australia
}

\begin{abstract}
Natural apatite samples were irradiated with $185 \mathrm{MeV} \mathrm{Au}$ and $2.3 \mathrm{GeV} \mathrm{Bi}$ ions to simulate fission tracks. The resulting track morphology was investigated using synchrotron small angle $\mathrm{x}$-ray scattering (SAXS) measurements before and after chemical etching. We present preliminary results from the SAXS measurement showing the etching process is highly anisotropic yielding faceted etch pits with a 6-fold symmetry. The measurements are a first step in gaining new insights into the correlation between etched and unetched fission tracks and the use of SAXS as a tool for studying etched tracks.
\end{abstract}

\section{Introduction}

Fission tracks are used for determining the age and thermal history of geological material by studying the number and length distribution of etched tracks that result from spontaneous fission of natural inclusions of uranium in the material [1]. The resulting high-energetic fission fragments produce narrow (a few nanometres) and long (up to ten of micrometres) cylindrical defects in the minerals [2] that show increased chemical etching compared to the undamaged material. The etching enlarges the original damage area such that it can be imaged using optical microscopy. When the sample being immersed in a suitable chemical solution, the solution will causes a preferential attack of the tracks thus enlarging the track radius. The currently used fission track dating techniques use empirical analysis of chemically etched track distributions, yet it largely ignores variations in un-etched tracks due to varying geological parameters such as pressure, temperature, crystallographic orientation and composition of the mineral.

To produce the tracks in the lab, we utilize the heavy ion accelerator facility available at the ANU and the UNILAC accelerator at GSI in Germany. The ANU accelerator is able to produce ions with the mass and energy corresponding to those of natural fission tracks, while the GSI machine is capable of producing tracks with higher energies, yet similar energy losses. At this facility it is also possible to generate ion tracks under high pressure and high temperature conditions.

Previous studies in our group focussed on the variations in un-etched track morphology characterised by synchrotron based small angle $\mathrm{x}$-ray scattering (SAXS) [3, 4]. SAXS is a non-destructive technique used to study the size, orientation and spatial arrangement of nano- to micro-sized objects. The applications cover various fields, for example the study of polymers in solution and in bulk, the shape of proteins, as well as a variety of nanosized materials. This method is ideally suited to study un-etched ion tracks with high precision as they are nearly identical, aligned, nano-sized objects [3]. In contrast to most other techniques it can assess the entire track and requires only a minimum of sample preparation [5].

The general aim of this work is to study how differences in the un-etched track morphology translate into etched ion track dimensions to bridge the gap between the fundamental research into track formation and their application in fission track dating. Etched tracks are studied using optical and scanning electron microscopy and the results directly correlated to those from un-etched tracks studied by SAXS. Furthermore the potential to measure etched track distributions using SAXS is exploited. Here, we present preliminary results from SAXS measurements on un-etched and etched tracks in Durango apatite.

\section{Experimental}

Natural apatite samples from Durango, Mexico have been used for this experiment. The chemical formula of apatite is $\mathrm{Ca}_{5} \mathrm{~F}\left(\mathrm{PO}_{4}\right)_{3}$ [3] with a hexagonal structure.

The sample was annealed at $450^{\circ} \mathrm{C}$ for 24 hours [6] to remove all natural tracks. After annealing, thin slices of

\footnotetext{
${ }^{\mathrm{a}}$ Corresponding author: allina.nadzri@anu.edu.au
} 
the crystal were prepared by cutting and polishing along different crystallographic orientations to thicknesses between 50 and $120 \mu \mathrm{m}$.

In order to form the tracks, apatite samples were irradiated with $185 \mathrm{MeV}$ Au ions using the ANU HeavyIon Accelerator facility and $2.3 \mathrm{GeV} \mathrm{Bi}$ ions at the UNILAC linear accelerator at the GSI Helmholtzcentre for Heavy Ion Research in Germany. Irradiation fluences of $10^{8} \mathrm{ions} / \mathrm{cm}^{2}$ and $10^{11} \mathrm{ions} / \mathrm{cm}^{2}$ were used to create well separated tracks. The samples with the lower fluence irradiation were chosen for the etching experiments to avoid overlap of the tracks after etching, as the tracks will enlarge significantly during chemical etching.

Following this, apatite samples irradiated with $10^{8}$ ions $/ \mathrm{cm}^{2}$ were chemically etched using diluted nitric acid $\left(\mathrm{HNO}_{3}\right)$ at room temperature. Two concentrations of the etchant were used: the commonly used 5 Molar (M) [710] and a lower concentration, $0.55 \mathrm{M}$. The lower concentration was used to achieve slower etching process hence reducing the timing errors between different etching experiments [6]. Each sample was etched up to 10 s followed by immersing the sample into deionized water to stop the etching process. The samples were then rinsed with acetone and ethanol and air-dried.

For characterization of the track radius, synchrotron based SAXS was performed at the Australian Synchrotron in Melbourne. The measurements were carried out using an x-ray energy of $11 \mathrm{keV}$ and camera lengths of 6.7 and $7.3 \mathrm{~m}$ for the etched samples and $0.9 \mathrm{~m}$ for the un-etched samples. The longer camera length was used to resolve the scattering into smaller angles of the larger etched tracks. SAXS experiments were performed in transmission mode and the images were collected with a Pilatus $1 \mathrm{M}$ detector with exposure times of 2, 5 and 10 s. Measurements were taken with the ion tracks aligned with and tilted by $5^{\circ}$ and $10^{\circ}$ with respect to the incoming beam. Additionally, un-irradiated samples were measured and the scattering signals were used as a reference for background removal. Experimental details for SAXS measurements of ion tracks can be found in references [3, $4]$.

\section{Results and Discussion}

\subsection{Latent (un-etched) tracks}

Figure 1 shows scattering images obtained from the SAXS measurement of un-etched Durango apatite irradiated with (a) $2.3 \mathrm{GeV}$ Bi and (b) $185 \mathrm{MeV} \mathrm{Au}$ ions with a fluence of $1 \times 10^{11}$ ions $/ \mathrm{cm}^{2}$. The ion tracks from the samples can be aligned such that the tracks axis is nearly parallel to the $\mathrm{x}$-ray beam. As a result, an isotropic scattering image can be seen as shown in figure 1(a) and (b) where the cross-section exhibits circular geometry. When the sample was tilted by $10^{\circ}$ with respect to the $\mathrm{x}$ ray beam, the scattering images become highly anisotropic with two narrow curved streaks visible in the pattern as shown in figure 1(c) and (d). These streaks are a consequence of the high aspect ratio of the ion tracks.
From the SAXS images, the scattering intensities can be extracted from the streaks and plotted as a function of the scattering vector $q$. The plots after background removal are shown in figure 2 . The strong oscillating scattering intensities yield information about the track radii and indicate monodisperse tracks with a sharp density change between the tracks and matrix material [3, $4,11]$. As reported in previous studies $[3,4,6,12]$, the most suitable model that fits the scattering intensity from ion tracks in Durango apatite is a cylinder model with a constant density. The solid lines in figure 2 represent the analytical fits to this model. A more detailed description of the model can be found in elsewhere $[13,14]$.
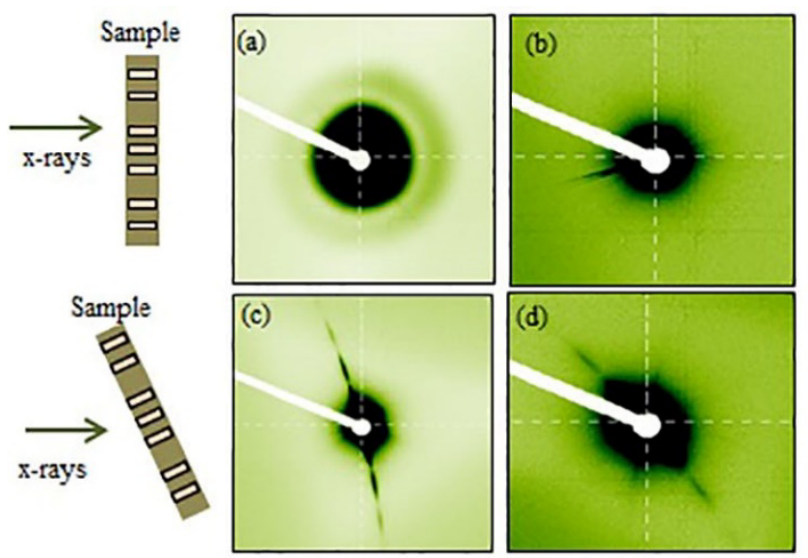

Figure 1. SAXS images of the unetched ion tracks in Durango apatite irradiated with a fluence of $1 \times 10^{11}$ ions $/ \mathrm{cm}^{2}$ by $2.3 \mathrm{GeV}$ Bi ions (a), (c), and $185 \mathrm{Mev} \mathrm{Au}$ ions (b), (d). (a) and (b) correspond to the ion tracks aligned and (c) and (d) to the tracks when tilted by $10^{\circ}$ with respect to the incoming x-ray beam.

From the fits in figure 2, the average latent track radius measured by SAXS for Durango apatite irradiated with $2.3 \mathrm{GeV} \mathrm{Bi}$ ions is $5.00 \pm 0.08 \mathrm{~nm}$ which consistent with the previous studies for apatite $[15,16]$. On the other hand, for the sample irradiated with $185 \mathrm{MeV}$, the track radius is $0.5 \mathrm{~nm}$ larger with a value of $5.50 \pm 0.12$ $\mathrm{nm}$ which also matches the result reported in [4]. This has been observed previously and explained by the so-called velocity effect [4]. The values of electronic energy loss $\mathrm{dE} / \mathrm{dx}$ for both irradiation conditions calculated from SRIM2008 [17] are $24 \mathrm{keV} / \mathrm{nm}$ for $185 \mathrm{Mev} \mathrm{Au}$ ions and $29.1 \mathrm{keV} / \mathrm{nm}$ for $2.3 \mathrm{Gev} \mathrm{Bi}$ ions respectively. It seems that these values are comparable, however, for a given energy loss, the ion with the lower velocity produces a higher density of created defects resulting in larger track radius [15]. 


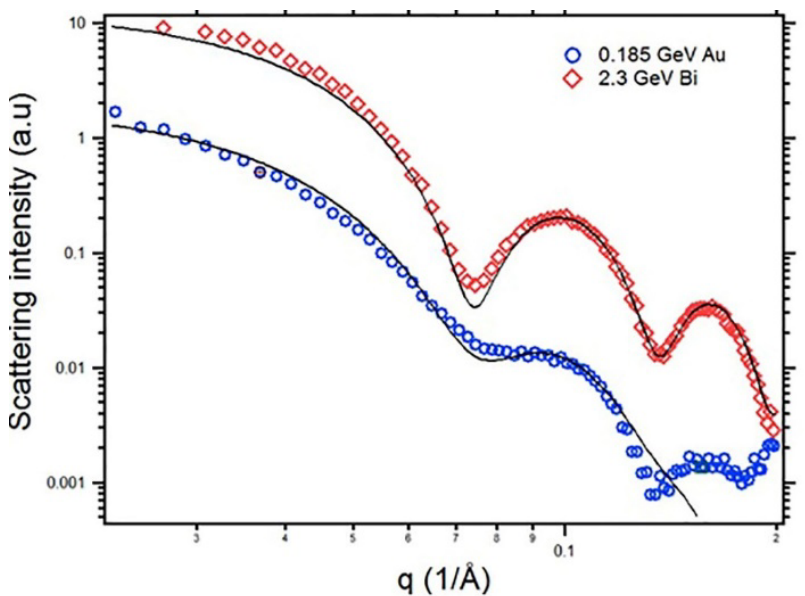

Figure 2. Scattering intensities as a function of the scattering vector $q$ from the SAXS data of unetched ion tracks in Durango apatite for the two irradiation conditions (open symbols). The solid lines are the corresponding fits using the hard cylinder model.

\subsection{Etched tracks}

The scattering images obtained from the SAXS measurements of etched tracks are presented in Figure 3. In figure 3 (a) and (c), the images represent the sample irradiated by $2.3 \mathrm{Gev}$ ions, etched using $0.55 \mathrm{M} \mathrm{HNO}_{3}$ for $10 \mathrm{~s}$ while figure 3 (b) and (d) corresponds to the sample irradiated by $185 \mathrm{MeV}$ ions, etched using $5 \mathrm{M}$ $\mathrm{HNO}_{3}$ for $4 \mathrm{~s}$. Significant differences to the un-etched tracks are apparent. While the un-etched tracks show isotropic images when aligned with the x-ray beam, after etching they exhibit hexagonal and 'star'-like pattersn with strong oscillations as apparent from figure 3 (a) and (b). This indicated an etching anisotropy in apatite yielding faceted etch pits with 6-fold symmetry. When tilted, both sample images reveal more 'streak' like patterns consistant with the tracks maintaining a high aspect ratio after etching. In contrast to the $2.3 \mathrm{GeV}$ ions irradiated sample, the sample irradiated with $185 \mathrm{MeV}$ ions looks more faceted with strong oscillation pattern. The reasons for this observation could include the different crystallographic orientation, different etching conditions and the ion and energy used to generate the tracks in apatite. Further data collection and analysis is required to determine exactly how these causes affect the scattering patterns and intensities.

The scattering intensities extracted from the streaks in figure 3 (a) are plotted in figure 4 . The inset in figure 4 shows the mask used for data extraction. The background subtraction was performed with the scattering intensity of an un-irradiated sample. The solid line represents the fit to the cylinder model identical to that used for the unetched tracks. While it appears that the etched tracks are facetted the fits to the cylinder model enable to estimate the etched track widths to $24.5 \pm 1.0 \mathrm{~nm}$. This analysis demonstrates that we can measure etched track radii very well using SAXS. However, the cylinder model was not able to fit the data from the $185 \mathrm{MeV}$ irradiated samples well. For this energy the tracks can be expected to be cone-shaped [6]. Further work is currently on going to find the suitable fitting for detailed data analysis including implementation of a cone form factor as well as systematic characterisation of the etched tracks as a function of the etching conditions $[3,4,16]$.

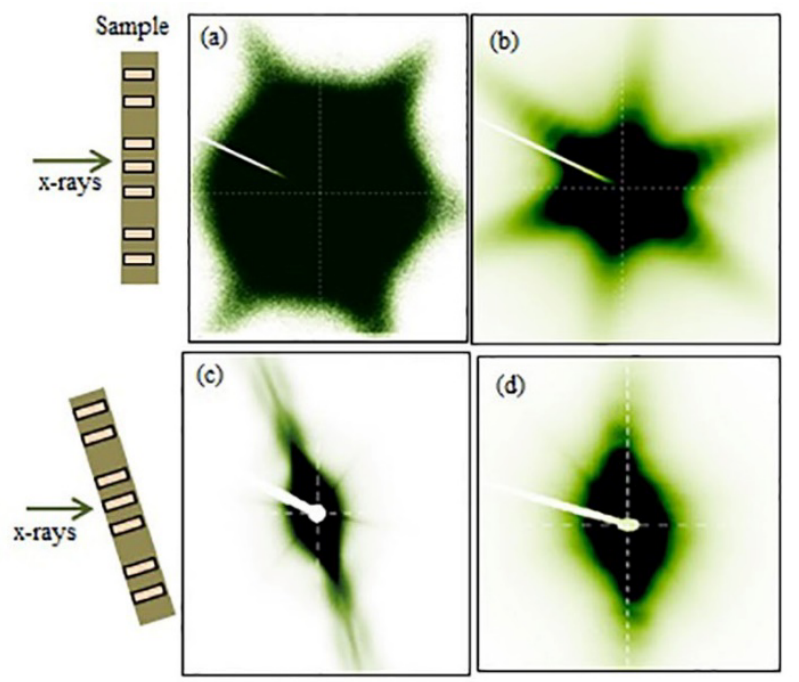

Figure 3. SAXS images of etched ion tracks in Durango apatite. In (a) the sample was irradiated by $2.3 \mathrm{GeV}$, etched for $10 \mathrm{~s}$ in $0.55 \mathrm{M} \mathrm{HNO}_{3}$ while in (b) the sample was irradiated by 185 $\mathrm{MeV}$, etched for $4 \mathrm{~s}$ with $5 \mathrm{M} \mathrm{HNO}_{3}$. Both samples were in the condition where the $\mathrm{x}$-ray beam parallel to the ions tracks. (c) and (d) are the ion tracks when tilted by $10^{\circ}$ with respect to the incoming x-rays beam.

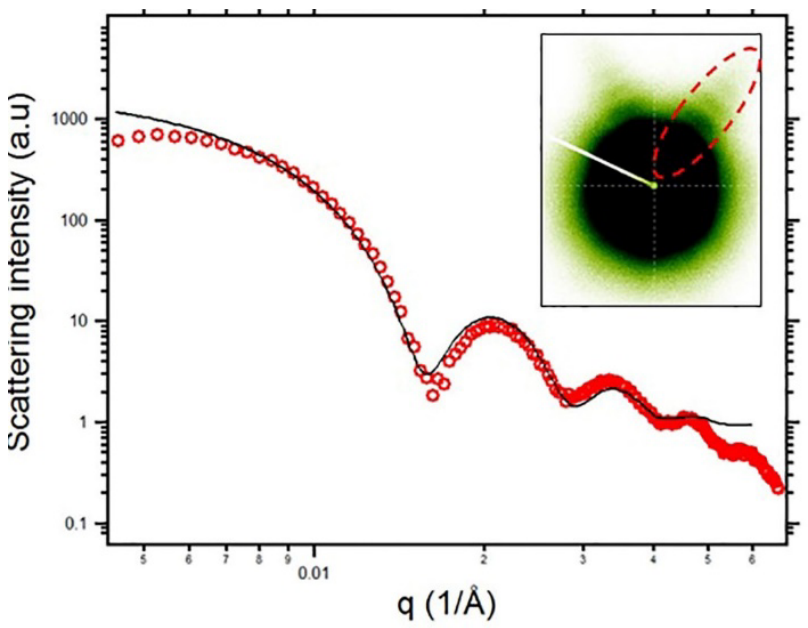

Figure 4. Scattering intensity after background removal from etched ion tracks in Durango apatite irradiated with $2.3 \mathrm{GeV}$ Bi ions, etched in $0.55 \mathrm{M} \mathrm{HNO}_{3}$ for 10s. The solid line is representing the fit to the hard cylinder model. The inset indicates the mask used to extract the scattering intensity plotted. 


\section{Conclusions}

In this investigation we characterized etched and unetched ion tracks using SAXS with the ultimate aim to establish the connection between the morphology of the two. The results from this investigation indicate that while the un-etched tracks show spherical cross-sections, the etching process is highly anisotropic and yields faceted etch pits with 6-fold symmetry. It was also shown that etched tracks generated by $2.3 \mathrm{GeV}$ Bi ions can be fitted analytically using the same hard cylinder model as used for un-etched tracks. Unlike in $2.3 \mathrm{GeV}$ irradiated sample, the hard cylinder model could not fit well the SAXS data from $185 \mathrm{MeV}$ irradiated sample. More work will need to be done to provide full details of the etched track morphology measured using SAXS. These results, while preliminary, suggest that SAXS can reveal details about the morphology of etched tracks in minerals and may help us to understand the correlation between etched and un-etched ion tracks in natural samples.

\section{Acknowledgements}

This research was undertaken on the SAXS/WAXS beamline at the Australian Synchrotron. P.K. acknowledges the Australian Research Council for financial support. A.N would like to acknowledge the Universiti Teknologi Mara (Malaysia) and Ministry of Higher Education, Malaysia for financial support.

\section{References}

1. R.L. Fleischer, P.B. Price and R.M. Walker, Nuclear tracks in solids: principles and applications. Univ of California Press (1975).

2. G.A. Wagner and P. Van den Haute, Fission Track Dating. Kluwer Academic Publishers: Dordrecht (1992).

3. B. Afra, M. Lang, M.D. Rodriguez, J. Zhang, R. Giulian, N. Kirby, R.C. Ewing, C. Trautmann, M. Toulemonde and P. Kluth. Phys. Rev. B. 83 (2011) 064116.

4. B. Afra, M.D. Rodriguez, M. Lang, R.C. Ewing, N. Kirby, C. Trautmann and P. Kluth. Nucl. Instrum. Methods Phys. Res. Sect. B. 286 (2012) 243.

5. H. Schnablegger and Y. Singh, A Practical Guide to SAXS: Getting acquainted with the principles. Austria: Anton Paar (2006).

6. J.M. Pray, The morphology of Etched and Unetched Ion Tracks in Apatite as a Function of Orientation and Thermal Annealing. Michigan: University of Michigan (2012).

7. W.D. Carlson, R.A. Donelick and R.A. Ketcham. American Mineralogist. 84 (1999) 1213.
8. R.A. Donelick, P.B. O'Sullivan and R.A. Ketcham. Reviews in Mineralogy and Geochemistry 58 (2005) 49.

9. R.A. Ketcham, R.A. Donelick and W.D. Carlson. American Mineralogist 84 (1999) 1235.

10. E. Sobel and D. Seward. Chemical Geology 271 (2010) 59.

11. D. Schauries, M. Lang, O. Pakarinen, S. Botis, B. Afra, M. Rodriguez, F. Djurabekova, K. Nordlund, D. Severin and M. Bender. J. Appl. Cryst. 46 (2013) 1558

12. P. Kluth, B. Afra, M.D. Rodriguez, M. Lang, C. Trautmann and R.C. Ewing. EPJ Web of Conferences 35 (2012) 03003.

13. B. Afra, M.D. Rodriguez, C. Trautmann, O.H. Pakarinen, F. Djurabekova, K. Nordlund, T. Bierschenk, R. Giulian, M.C. Ridgway, G. Rizza, N. Kirby, M. Toulemonde and P. Kluth. J. Phys. Condens. Matter 25 (2013) 045006.

14. B. Kuttich, M. Engel, C. Trautmann and B. Stühn. Applied Physics A 114 (2014) 387.

15. B. Afra, Investigation of ion-track morphology and annealing behavior using small-angle X-ray scattering. Canberra: Australian National University (2013).

16. D. Schauries, B. Afra, T. Bierschenk, M. Lang, M. Rodriguez, C. Trautmann, W. Li, R. Ewing and P. Kluth. Nucl. Instrum. Methods Phys. Res. Sect. B 326 (2014) 117.

17. J.F. Ziegler, M.D. Ziegler and J.P. Biersack. Nucl. Instrum. Methods Phys. Res. Sect. B 268 (2010) 1818. 